\title{
Relation of morning serum cortisol to prothrombotic activity in women with stable coronary artery disease
}

\author{
Roland von Känel · Brent T. Mausbach • \\ Brigitte M. Kudielka $\cdot$ Kristina Orth-Gomér
}

Published online: 12 June 2007

(C) Springer Science+Business Media, LLC 2007

\begin{abstract}
Background Increased circulating cortisol levels have been associated with severity of atherosclerosis. Low-grade systemic thrombogenicity plays a major role in the initiation and progression of coronary disease. We hypothesized a direct relationship between cortisol and hemostasis factors related to a prothrombotic state in coronary artery disease.

Methods We measured morning serum cortisol and activated clotting factor VII, fibrinogen, von Willebrand factor antigen, and plasminogen activator inhibitor-1 activity in 285 women ( $56 \pm 7$ years) between 3 and 6 months after an acute coronary event. To test whether the relationship between cortisol and hemostasis factors would be independent, statistical adjustment was made for demographic, biomedical, life style, and psychosocial variables.
\end{abstract}

R. von Känel $(\bowtie)$

Department of General Internal Medicine, University Hospital,

Berne 3010, Switzerland

e-mail: roland.vonkaenel@insel.ch

R. von Känel

Cardiovascular Prevention and Rehabilitation, Swiss

Cardiovascular Center Berne, University Hospital, Berne,

Switzerland

B. T. Mausbach

Department of Psychiatry, University of California, San Diego, USA

B. M. Kudielka

Department of Theoretical and Clinical Psychobiology,

University of Trier, Trier, Germany

K. Orth-Gomér

Division of Preventive Medicine, Department of Public Health

Sciences, Karolinska Institute, Stockholm, Sweden
Results Higher serum cortisol levels predicted higher fibrinogen $(\beta=.17, P=.001)$ and higher von Willebrand factor $(\beta=.16, P=.008)$, all independently of covariates, including $\mathrm{C}$-reactive protein, which was also an independent predictor of fibrinogen $(\beta=.20, P=.001)$ and von Willebrand factor $(\beta=.16, P=.004)$. Higher levels of vital exhaustion were associated with higher levels of activated clotting factor VII independently of covariates and depression $(\beta=.18, P=.045)$. Cortisol showed crude correlations with vital exhaustion $(r=.14$, $P=.022)$ and with depression $(r=.13, P=.043)$ but did not mediate the relationship between psychosocial variables and hemostatic factors.

Conclusions Morning serum cortisol showed a modest but independent association with prothrombotic activity in women with coronary artery disease suggesting that increased cortisol levels might contribute to atherosclerosis via eliciting a hypercoagulable state.

Keywords Cortisol - Cardiovascular disease . Hemostasis · Inflammation

\section{Introduction}

An exaggerated physiological stress response to a variety of environmental stimuli and as mediated by the hypothalamic-pituitary adrenal (HPA) axis may contribute to cardiovascular disease $[1,2]$. During the first hours of an acute myocardial infarction (AMI), hyperactivity of the HPA axis is evident as regards cortisol spillover [3-5]. Higher cortisol levels in AMI patients at hospital admission predicted extent of myocardial damage [5, 6], risk of recurrent non-fatal MI [7], and mortality [6]. Cortisol excess may also exert cardiovascular peril in patients with 
stable coronary artery disease (CAD) who showed more severe coronary sclerosis with higher circulating cortisol levels [8-10].

The mechanisms through which circulating cortisol contributes to atherosclerosis are likely manifold. HPA axis dysregulation is associated with cardiovascular disease risk factors clustering in the metabolic syndrome such as truncal obesity, dyslipidemia, and high blood pressure [11]. A prothrombotic state, as characterized by elevated circulating fibrinogen and plasminogen activator inhibitor (PAI)-1, also belongs to the metabolic disturbances pertinent to cardiovascular disease [12]. However, the relationship between cortisol and low-grade systemic thrombogenicity playing a major role in the pathogenesis of atherosclerosis and onset of acute ischemic events [13] has not been investigated in CAD patients.

CAD is under-researched in women relative to men [14]. In this study, we investigated female patients because of a particular paucity of data on women's psychosocial and other cardiovascular risk factors, including hemostatic measures. We studied a relatively young group of women coronary patients because their ailments can be viewed as premature, and not as a sign or symptom of the ordinary ageing process. We hypothesized that morning cortisol levels would be associated with a prothrombotic state, as defined by increased plasma levels of four hemostasis factors, i.e. activated clotting factor VII (FVIIa), fibrinogen, von Willebrand factor (VWF), and PAI-1 in women with CAD. The reasoning for this hypothesis was threefold. First, all of these factors independently predicted coronary events in prospective large-scale epidemiological studies [15-16]. Second, patients with Cushing's disease confer a prothrombotic state characterized by high levels of fibrinogen, VWF, and PAI-1 [17-19]. This hypercoagulability is thought to be a consequence of excessive endogenous cortisol production and to increase cardiovascular risk of Cushing patients [20]. Third, exogenous glucocorticoid administration increases procoagulant activity (e.g. FVII, fibrinogen, and VWF) and dampens fibrinolytic activity in humans [21-24]. We further investigated whether the relationship between cortisol and hemostasis factors is independent of demographic, medical, and life style covariates commonly affecting hemostatic function. Because hemostasis and inflammation are highly interacting in promoting the atherosclerotic process [25], we particularly aimed to investigate whether the cortisolhemostasis relationship is independent of low-grade proinflammatory activity as measured by serum levels of C-reactive protein (CRP).

Basal cortisol levels in stable CAD are unlikely influenced by the acute phase of MI because cortisol rise during AMI usually returns to normal within one week post-MI [4, 26]. In stable CAD, possible correlates of elevated basal cortisol levels are depressed mood [27] and, as previously shown in the sample of women patients investigated in this study, feelings of vital exhaustion [9]. Depression and vital exhaustion are both prevalent in patients with CAD and are independent CAD risk factors [28]. Depressed individuals have demonstrated elevated levels of fibrinogen, FVII, and PAI-1 [29-31]. Similarly, exhausted subjects show increased levels of fibrinogen and PAI-1 [32, 33]. We therefore also investigated whether cortisol relates to hemostasis factors independent of depression and vital exhaustion or, alternatively, whether cortisol is a mediator of the relationship between hemostasis and psychosocial factors.

\section{Materials and methods}

\section{Patients and study design}

The Ethics Committee of the Karolinska Institutet approved the study protocol and all subjects provided informed consent. As previously described in more detail [34], we included women patients aged $\leq 65$ years who were hospitalized for AMI or unstable angina pectoris (UAP) during a 3-year period between 1991 and 1994. The diagnosis of AMI was made following WHO criteria [35] of typical chest pain, cardiac enzyme patterns, and/or diagnostic ECG changes as classified by the Minnesota code [36]. UAP was defined as new onset of severe AP, or deterioration of known stable AP in the 4 weeks before hospital admission [37]. A total of 292 women (110 AMI, 182 UAP) were enrolled and examined at the research clinic as outpatients between 3 and 6 months after discharge from the hospital. At this time, they were considered to be in a stable cardiac and metabolic condition and without acute phase reaction influencing laboratory measurements, including hemostasis factors and CRP. For the present study, we performed analyses on 285 women with an acute coronary event who had complete data in terms of cortisol levels, CRP, and at least one of the four hemostasis factors measured. Although not formally assessed, there was not much clinical co-morbidity in this relatively young group of women patients.

\section{Demographic and health characteristics}

Detailed assessment of demographic, medical, and lifestyle measures has previously been described [34]. Menopausal status, body mass index (BMI) and blood pressure (BP) were determined by standard methods. Mean BP was computed by the formula $(2 / 3 *$ diastolic $\mathrm{BP})+(1 / 3 *$ systolic BP). Medication (aspirin, oral anticoagulants) was abstracted from hospital charts and verified upon arrival at 
the research clinic. Smoking habits, physical activity during leisure time categorized as sedentary or active, and daily alcohol intake were assessed by standard questionnaires.

\section{Psychosocial measures}

Vital exhaustion was measured by means of an early version of the Maastricht Questionnaire [38] that was predictive for first-time MI in women [39] and new cardiac events in men after percutaneous transluminal coronary angioplasty [40]. The exhaustion scale comprises 19 items scored with 1 ("no"), 2 ("don't know"), or 3 ("yes") and essentially asks about unusual fatigue, increased irritability, and feelings of demoralization. Items are summed to create a total exhaustion score, with higher scores referring to higher levels of exhaustion (range 19 to 57). In 32 patients, missing items were substituted by the person's mean of all completed items. Data imputation was not performed in four patients who had less than half of items present [41] yielding a total of 281 patients with a complete vital exhaustion sum score for analyses.

Depressive symptoms were measured by means of a previously published 10-item questionnaire [42] of which we excluded the item asking about sexual activity to potentially increase response rate. The current version thus included 9 items with yes and no answers as alternatives. The yes answers were summed with a high score indicating high degree of depressive symptom level (range 0 to 9 ). Of the 285 patients, 23 failed to adequately complete the questionnaire (i.e., answered fewer than 5 items). We imputed scores for an additional 25 patients who had at least five items present [41], yielding 262 patients with a complete depression sum score for analyses.

Although vital exhaustion and depression share conceptual similarities like lack of energy, increased irritability, and demoralization, the two constructs show also some important differences [28]. For instance, sadness, guilt, and feelings of worthlessness are characteristics of depression and do not belong to the definition criteria of exhaustion [43]. Applying factorial analyses we previously showed that depression and vital exhaustion constitute distinct psychological concepts in healthy individuals [44].

\section{Laboratory analyses}

After an overnight fast and 5-min supine rest, blood samples were obtained between 8 am and $10 \mathrm{am}$, when it was assumed that the cortisol awakening rise had declined [45]. For determining hemostatic factors, venous blood was drawn without stasis by antecubital, direct venipuncture into vacuum tubes containing $0.13 \mathrm{~mol} / \mathrm{l}$ trisodium citrate
(9:1 blood/citrate, vol/vol). Samples were immediately centrifuged at room temperature at $2,000 \times g$ for $15 \mathrm{~min}$ and plasma was stored at $-70{ }^{\circ} \mathrm{C}$ until further analyses. FVIIa was determined by a clotting assay using soluble recombinant truncated tissue factor (a gift from Professor James H. Morrissey, University of Illinois at UrbanaChampaign, Urbana, IL) [46]. Fibrinogen was measured by a polymerization rate method [47]. VWF:Ag was analyzed by an enzyme-linked immunosorbent assay (Asserachrom Stago, Asnières, France). PAI-1 activity was determined by a functional spectrophotometric method (Biopool $\mathrm{AB}$, Umea, Sweden). Inter-assay and intra-assay coefficients of variation $(\mathrm{CV})$ were $4 \%$ for fibrinogen, $2 \%$ for vWF:Ag, $<10 \%$ for FVIIa, and $<12 \%$ for PAI- 1 activity.

For the determination of serum total cholesterol, glucose, CRP, and cortisol, fasting venous blood was drawn into serum-separated tubes and centrifuged at 3,000 $\times g$ for $10 \mathrm{~min}$; serum was frozen and stored at $-70{ }^{\circ} \mathrm{C}$. Cholesterol was determined using CHOD-PAP enzymatic methods with reagents from Boehringer Mannheim (Germany) and measured with an automated multichannel analyzer [48]. Glucose was analyzed by the GOD-PAP method [49]. CRP was determined using a turbidimetric immunoassay, standardized against WHO, yielding values between $1-120 \mathrm{mg} / \mathrm{L}(\mathrm{CVs} \leq 12 \%)$. Cortisol was measured by standardized radioimmunoassay [50]. All cortisol samples were analysed in duplicate and mean values were computed; inter- and intra-assay CVs were $\leq 20 \%$ and $\leq 10 \%$, respectively [51].

\section{Statistical analysis}

Data were analysed using SPSS 13.0 software (SPSS Inc., Chicago, IL, USA). All testing was two-tailed with $P \leq .05$. Because of a non-normal distribution (Kolmogorov-Smirnov Test), FVII, fibrinogen, and VWF values were logarithmically transformed and PAI-1 values were square-root transformed. For glucose, CRP, cortisol, and depression values we applied Blom normal score transformation because neither logarithmic nor square root transformation yielded a normal distribution of these measures. For clarity, figures show original cortisol values. Pearson correlations quantified the bivariate relationship between two variables.

To investigate the predictive value of cortisol for levels of hemostasis measures, we conducted hierarchical linear regression analyses. In these analyses, we entered in Block 1 thirteen a priori covariates potentially affecting hemostasis, namely age, index event, menopausal status, medication (aspirin, oral anticoagulants), lifestyle factors (smoking status, physical activity, alcohol consumption), metabolic factors (BMI, cholesterol, mean BP, glucose), and CRP. Cortisol was entered in Block 2. In a next set of 
analyses, psychosocial factors (vital exhaustion, depression) were entered separately and together in Block 2 to test whether these would affect the relationship between cortisol levels and hemostasis measures. In the case of statistically significant effects, we also computed effect size measure (Cohen's $f^{2}$ ) to provide an estimate of the clinical significance of these relationships. By convention, $f^{2}$ effect sizes of $0.02,0.15$, and 0.35 are considered small, medium, and large, respectively [52].

To statistically account for multiple comparisons between cortisol levels and four hemostasis factors, we applied Bonferroni correction with significance level set at $P<.013$. We used the procedures described by Baron and Kenny [53] to test the potential mediating role of cortisol on the relations between psychosocial and hemostatic factors.

\section{Results}

Subjects' characteristics

The demographic, health, and laboratory characteristics of the 285 female patients with stable CAD, the distribution of these variables, and occasionally missing data are summarized in Table 1.

Bivariate correlation analysis

\section{Laboratory measurements}

Except from the relationship between FVII and VWF, all hemostatic factors showed positive correlations with each other (r's between .13 and .40, p's between .031 and
Table 1 Characteristics of 285 women with coronary artery disease

Data were missing for menopausal status in three subjects $(1 \%)$, for physical activity in four subjects (1\%), and for alcohol consumption in 11 subjects $(4 \%)$

\begin{tabular}{|c|c|c|c|c|c|c|}
\hline & $\mathrm{N}$ & $\%$ & Mean & SD & Median & Range \\
\hline Age (years) & 285 & & 56.0 & 7.0 & 57.0 & $30-66$ \\
\hline \multicolumn{7}{|l|}{ Index event } \\
\hline Acute myocardial infarction & 107 & $37 \%$ & & & & \\
\hline Unstable angina pectoris & 178 & $63 \%$ & & & & \\
\hline \multicolumn{7}{|l|}{ Menopausal status } \\
\hline Premenopausal & 63 & 22 & & & & \\
\hline Postmenopausal with HRT & 33 & 12 & & & & \\
\hline Postmenopausal without HRT & 186 & 65 & & & & \\
\hline Aspirin & 200 & 70 & & & & \\
\hline Oral anticoagulants & 16 & 6 & & & & \\
\hline \multicolumn{7}{|l|}{ Smoking status } \\
\hline Current & 58 & 20 & & & & \\
\hline Former & 137 & 48 & & & & \\
\hline Never & 90 & 32 & & & & \\
\hline \multicolumn{7}{|l|}{ Physical activity } \\
\hline Sedentary & 68 & 24 & & & & \\
\hline Active & 213 & 75 & & & & \\
\hline \multicolumn{7}{|l|}{ Alcohol consumption (g/d) } \\
\hline$\leq 5 \mathrm{~g} / \mathrm{d}$ (light drinker) & 194 & 68 & & & & \\
\hline$>5 \mathrm{~g} / \mathrm{d}$ (moderate drinker) & 80 & 28 & & & & \\
\hline Body mass index $\left(\mathrm{kg} / \mathrm{m}^{2}\right)$ & 285 & & 27.0 & 4.4 & 26.6 & $18.6-42.3$ \\
\hline Total serum cholesterol $(\mathrm{mmol} / \mathrm{l})$ & 285 & & 6.5 & 1.2 & 6.4 & $3.2-11.4$ \\
\hline Fasting serum glucose $(\mathrm{nmol} / \mathrm{l})$ & 285 & & 5.8 & 3.3 & 4.9 & $2.9-21.9$ \\
\hline Mean blood pressure (mmHg) & 284 & & 91.5 & 12.5 & 89.7 & $64.7-134.3$ \\
\hline Serum C-reactive protein $(\mathrm{mg} / \mathrm{l})$ & 285 & & 3.8 & 5.4 & 3.0 & $1.0-57.0$ \\
\hline Morning serum cortisol (nmol/l) & 285 & & 170.2 & 45.4 & 162.0 & $93.0-303.0$ \\
\hline Vital exhaustion score & 281 & & 39.1 & 8.5 & 39.0 & $19.0-57.0$ \\
\hline Depression score & 262 & & 3.9 & 2.9 & 4.0 & $0-9.0$ \\
\hline Activated FVII (ng/ml) & 272 & & 1.29 & 0.34 & 1.28 & $0.44-2.42$ \\
\hline Fibrinogen $(\mathrm{g} / \mathrm{l})$ & 285 & & 3.66 & 0.81 & 3.50 & $1.40-7.20$ \\
\hline VWF antigen (IU/ml) & 285 & & 1.50 & 0.76 & 1.31 & $0.62-7.32$ \\
\hline PAI-1 activity (IU/ml) & 285 & & 15.0 & 14.2 & 11.0 & 0-87.0 \\
\hline
\end{tabular}



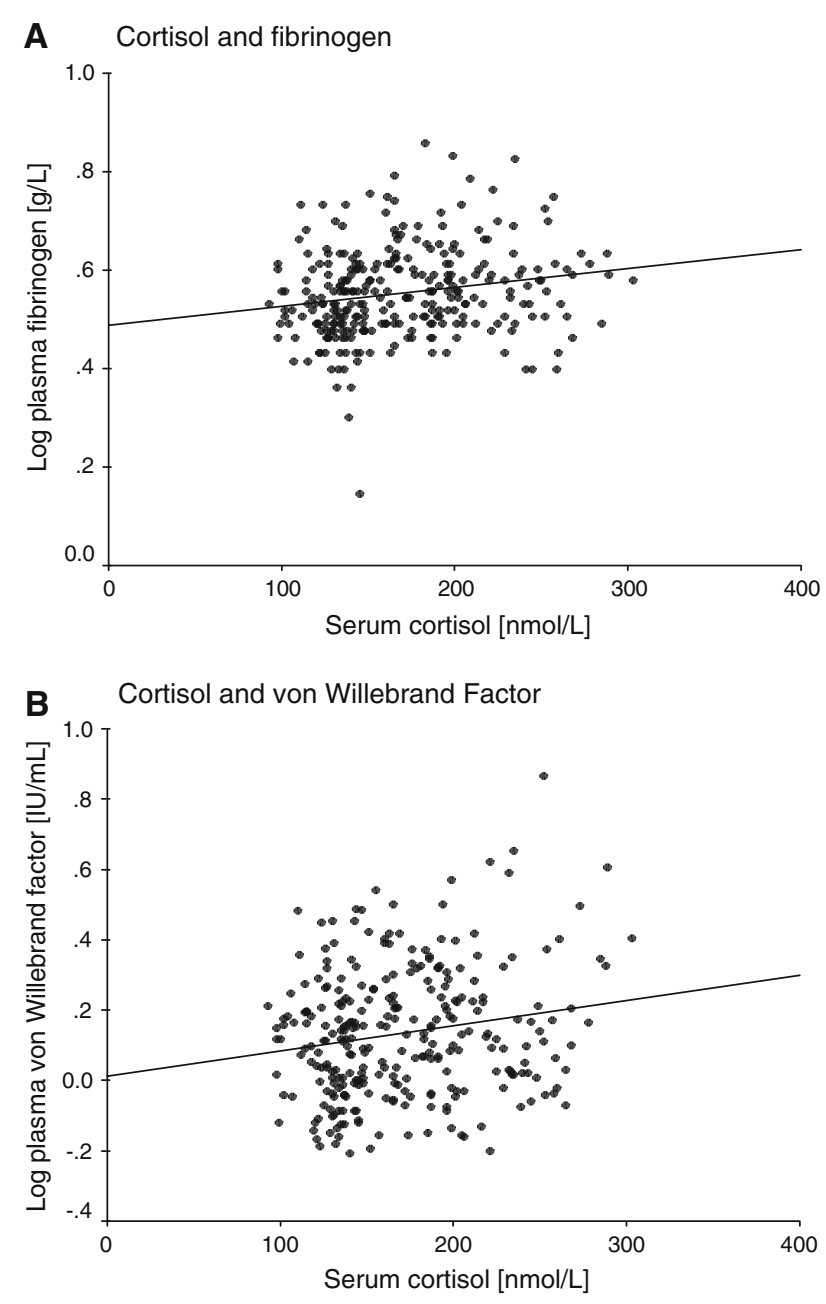

Fig. 1 Higher cortisol levels correlated significantly with higher levels of fibrinogen $(r=.19, n=285$; Panel A) and with higher levels of VWF antigen $(r=.17, n=285$; Panel $\mathbf{B})$

<.001). Cortisol correlated with fibrinogen $(r=.19$, $p=.002$; Fig. 1, Panel A) and with VWF $(r=.17$, $P=.004$; Fig. 1, Panel B) but not significantly so with FVII and PAI-1. CRP showed significant associations with fibrinogen $(r=.27, P<.001)$, with VWF $(r=.17$, $P=.004)$, and with PAI-1 $(r=.16, P=.009)$ but not with FVII and cortisol.

\section{Psychosocial factors}

Cortisol showed a positive relationship with total scores of vital exhaustion $(r=.14, P=.022)$ and of depressed mood $(r=.13, P=.043)$. In contrast, psychosocial factors were not significantly correlated with any hemostatic factor and with CRP. Because mediational tests require significant relationships between the independent and dependent variables, cortisol was no longer considered a mediator of the relations between psychosocial and hemostatic factors, although we continued to control for psychosocial factors in our multi-variate models.

Hierarchical linear regression analysis

\section{Predictors of hemostatic factors}

Table 2 shows results of the four regression models with adjustment made for 13 covariates in one block. All models were significant explaining between $18 \%$ and $35 \%$ of the variance in the respective hemostatic factor.

There were several independent relationships between hemostatic factors and life style variables and metabolic indices. Lower levels of physical activity and of alcohol consumption were associated with higher levels of fibrinogen and VWF. Metabolic factors were directly associated with higher FVII and PAI-1 values.

The significant relationships between hemostatic factors and CRP and cortisol, respectively, were independent of all covariates and also of each other. Higher CRP levels predicted higher fibrinogen levels $\left(P=.001 ; f^{2}=0.04\right)$ and higher VWF levels $\left(P=.009 ; f^{2}=0.03\right)$. Higher cortisol levels also predicted higher levels of fibrinogen $(P=.004$; $\left.f^{2}=0.03\right)$ and of VWF $\left(P=.008 ; f^{2}=0.02\right)$.

\section{Role of psychosocial factors}

Next we tested whether considering psychosocial factors in models would affect the independent relationships between hemostatic factors and CRP and cortisol, respectively. However, significance levels of these relationships were maintained when exhaustion and depression were entered into equations either separately or together in Block 2 (data not shown in detail).

Vital exhaustion emerged as an independent predictor of FVII when controlling for depression $(\beta=.18, P=.045$; $\left.f^{2}=0.02\right)$, whereas exhaustion and depression were not independent predictors of fibrinogen, VWF, and PAI-1.

\section{Discussion}

We found evidence for our primary hypothesis of a relationship between circulating cortisol levels and thrombogenic activity. We showed that morning serum cortisol levels are directly associated with plasma levels of fibrinogen and VWF compatible with the notion that HPA axis activity directly relates to a prothrombotic state in women with stable CAD. At the time when we conducted our study, one-time determination of cortisol in the morning was a common method to assess HPA axis functioning, whereas measurement of diurnal cortisol activity has now become the standard procedure for this purpose [54]. 
Table 2 Regression model for hemostasis measures
The colums show standardized $\beta$-coefficients for the independent relationships between covariates and hemostasis measures with significance levels: $* P \leq .05$, $* * P \leq .01, * * * P \leq .001 \mathrm{VWF}$, von Willebrand factor; PAI-1, plasminogen activator inhibitor-1

\begin{tabular}{lllll}
\hline Covariates entered & \multicolumn{2}{l}{ Hemostatic factor } & & PAI-1 \\
\cline { 2 - 5 } & Factor VII & Fibrinogen & VWF & $-.17^{*}$ \\
\hline Age & .03 & .02 & .11 & $<.01$ \\
Index event & -.07 & .11 & $.15^{*}$ & .03 \\
Menopausal status & -.03 & .04 & .06 & -.03 \\
Aspirin & .05 & $<-.01$ & -.03 & -.03 \\
Oral anticoagulants & $-.25^{* * *}$ & .09 & $<.01$ & $.12^{*}$ \\
Smoking status & $<-.01$ & .09 & .06 & .03 \\
Physical activity & -.03 & $-.12^{*}$ & $-.13^{*}$ & -.03 \\
Alcohol use & -.03 & $-.14^{*}$ & $-.15^{*}$ & $.37^{* * *}$ \\
Body mass index & $.18^{* *}$ & .11 & .08 & $.15^{* *}$ \\
Total cholesterol & $.25^{* * *}$ & $.17^{* *}$ & .11 & $<-.01$ \\
Mean blood pressure & -.01 & .02 & .04 & $.27^{* * *}$ \\
Glucose & .08 & .01 & -.05 & .08 \\
C-reactive protein & .06 & $.20^{* * *}$ & $.16^{* *}$ & .04 \\
Cortisol & -.09 & $.17^{* *}$ & $.16^{* *}$ & $\mathrm{~F}_{14,250}=9.6$ \\
Df & $\mathrm{F}_{14,237}=3.9$ & $\mathrm{~F}_{14,250}=5.2$ & $\mathrm{~F}_{14,250}=3.9$ & $R^{2}=.35$, \\
Variance explained by & $R^{2}=.19$, & $R^{2}=.23$, & $R^{2}=.18$, & $P<.001$ \\
the entire model & $P<.001$ & $P<.001$ & $P<.001$ &
\end{tabular}

Nonetheless, our findings appear to be robust because the association with hemostatic factors was seen with one single measurement of morning cortisol. The relationship between cortisol and hemostatic factors maintained significance even when controlling for a reasonable set of correlates of hemostatic function. Importantly, although CRP was independently associated with fibrinogen and VWF in its own right, adjustment for CRP as a proxy measure of systemic inflammation did not affect the relationship between cortisol and hemostasis.

Contrary to our primary hypothesis, FVII and PAI-1 were unrelated to cortisol levels. Metabolic factors predicted PAI-1 and FVII apparently stronger than did cortisol. Unique variances in hemostasis factors explained by cortisol were rather modest as compared to variances explained by BMI, total cholesterol, and glucose, respectively. Some authors indeed advocate that PAI-1 is to be viewed as a cardiovascular risk factor only because of its strong relation to the metabolic syndrome [55]. In line with this reasoning, we previously showed that the direct association between overnight cortisol excretion in urine and morning PAI-1 levels became nonsignificant particularly when controlling for metabolic factors [56].

Metabolic effects of circulating cortisol on the hemostatic system could be of clinical importance. Recent metaanalyses suggest that the predictive value of hemostatic factors for cardiovascular events is most robust in terms of fibrinogen [15] and VWF [16] both associated with serum cortisol in our patients. Our study is unable to prove a causal relationship between cortisol and fibrinogen and VWF, respectively, in CAD patients which only a longitudinal design could substantiate if cortisol and hemostatic factor levels turned out to covary over time. In spite of its cross-sectional nature, our study is compatible with basic research showing that glucocorticoids enhance production of hemostatic factors on the cellular and protein level. Glucocorticoids induced fibrinogen-mRNA expression in cultured hepatocytes [57] and VWF-mRNA expression in cultured human umbilical endothelial cells [24], respectively. Moreover, exogenous administration of therapeutic dosages of dexamethasone for a few days resulted in increased plasma levels of fibrinogen [21] and of VWF [23]. Such procoagulatory mechanisms might help explain the previously observed relationship between higher circulating cortisol levels and more severe atherosclerosis, including in our female patients [8, 9], and between a greater awakening cortisol response and atherosclerosis progression in women [58].

Vital exhaustion and depression did not affect the relationship between cortisol and hemostatic factors. However, we found that vital exhaustion was an independent predictor of FVII. The latter is a novel finding that adds to the growing literature on a prothrombotic state in subjects who are exhausted [28, 32, 33]. The relationship between exhaustion and FVII was not mediated by cortisol suggesting that other stress-mediating systems might be involved [1].

We acknowledge the following limitations of our study. Accurate assessment of HPA axis activity and of CRP was compromised by our methodology. Morning cortisol levels are influenced by a variety of factors and do not necessarily reflect the diurnal course of HPA axis activity which 
requires repeated cortisol measurements throughout the day [54]. We were able to control for factors like age, menopausal state and life style but we could not control for others, particularly for the awakening time influencing morning cortisol rise [59]. It therefore would be important to replicate our hemostatic findings with strict reference to the time of awakening and in relation to diurnal cortisol activity. Moreover, we did not use a high-sensitive assay to measure CRP which is the gold standard in nowadays cardiovascular risk assessment [60]. Although statistically significant, effect sizes of the relationships between cortisol and VWF and fibrinogen on the one hand and between vital exhaustion and FVIIa on the other were small. It therefore remains to be seen whether these associations are of clinical importance. We investigated women with stable CAD when the acute physiological stress response to the event was assumed to have abated. We therefore are unable to state to which extent cortisol would relate to hemostatic activity in the early phase of an AMI when cortisol levels are highest. We also do not know whether the relationship between cortisol and hemostasis will sustain for longer than half-a-year after the event. This seems important as it can be assumed that the longer this relationship is maintained the greater the harm exerted on coronary arteries in the long run. Caution is needed in generalizing our findings across gender because our data could or could not be unique to women with CAD. Future studies may also want to investigate whether the relationship between endogenous cortisol levels and prothrombotic activity is specific to CAD populations or can also be observed in individuals free of cardiovascular disease.

Taken together, our findings suggest that cortisol levels could contribute to prothrombotic activity in women with stable CAD providing one explanation for the empirical observation relating circulating cortisol to atherosclerotic vessel damage.

Acknowledgements This study was supported by awards to Professor Kristina Orth-Gomér from the National Institutes of Health (RO1HL45785-02), the Swedish Science Foundation (521-2005-6921), and the Bank of Sweden Tencentenary Fund (2000-0349-01-02).

\section{References}

1. McEwen BS (1998) Protective and damaging effects of stress mediators. N Engl J Med 338:171-179

2. Sapolsky RM, Romero LM, Munck AU (2000) How do glucocorticoids influence stress responses? Integrating permissive, suppressive, stimulatory, and preparative actions. Endocr Rev 21:55-89

3. Wiener K (1977) Plasma cortisol, corticosterone and urea in acute myocardial infarction: clinical and biochemical correlations. Clin Chim Acta 76:243-250
4. Donald RA, Crozier IG, Foy SG, et al. (1994) Plasma corticotrophin releasing hormone, vasopressin, ACTH and cortisol responses to acute myocardial infarction. Clin Endocrinol 40:499-504

5. Subbs PJ, Laycock J, Alaghband-Zadeh J, Carter G, Noble MI (1999) Circulating stress hormone and insulin concentrations in acute coronary syndromes: identification of insulin resistance on admission. Clin Sci 96:589-595

6. Bain RJ, Fox JP, Jagger J, Davies MK, Littler WA, Murray RG (1992) Serum cortisol levels predict infarct size and patient mortality. Int J Cardiol 37:145-150

7. Tenerz A, Nilsson G, Forberg R, et al. (2003) Basal glucometabolic status has an impact on long-term prognosis following an acute myocardial infarction in non-diabetic patients. J Intern Med 254:494-503

8. Troxler RG, Sprague EA, Albanese RA, Fuchs R, Thompson AJ (1977) The association of elevated plasma cortisol and early atherosclerosis as demonstrated by coronary angiography. Atherosclerosis 26:151-162

9. Koertge J, Al-Khalili F, Ahnve S, Janszky I, Svane B, SchenckGustafsson K (2002) Cortisoland vital exhaustion in relation to significant coronary artery stenosis in middle-aged women with acute coronary syndrome. Psychoneuroendocrinology 27: 893-906

10. Matthews K, Schwartz J, Cohen S, Seeman T (2006) Diurnal cortisol decline is related to coronary calcification: CARDIA study. Psychosom Med 68:657-661

11. Lett HS, Blumenthal JA, Babyak MA, et al. (2004) Depression as a risk factor for coronary artery disease: evidence, mechanisms, and treatment. Psychosom Med 66:305-315

12. Grundy SM, Brewer HB Jr, Cleeman JI, Smith SC Jr, Lenfant C (2004) Definition of metabolic syndrome: Report of the National Heart, Lung, and Blood Institute/American Heart Association conference on scientific issues related to definition. Circulation 109:433-438

13. Shah PK (2006) Thrombogenic risk factors for atherothrombosis. Rev Cardiovasc Med 7:10-16

14. Rosenfeld AG (2006) State of the heart: building science to improve women's cardiovascular health. Am J Crit Care 15: $556-566$

15. Fibrinogen Studies Collaboration (2005) Plasma fibrinogen level and the risk of major cardiovascular diseases and nonvascular mortality: an individual participant meta analysis. JAMA 294:1799-1809

16. Danesh J. Wheeler JG, Hirschfield GM, et al. (2004) C-reactive protein and other circulating markers of inflammation in the prediction of coronary heart disease. N Engl J Med 350:13871397

17. Colao A, Pivonello R, Spiezia S, et al. (1999) Persistence of increased cardiovascular risk inpatients with Cushing's disease after five years of successful cure. J Clin Endocrinol Metab 84:2664-2672

18. Casonato A, Pontara E, Boscaro M, et al. (1999) Abnormalities of von Willebrand factor arealso part of the prothrombotic state of Cushing's syndrome. Blood Coagul Fibrinolysis 10:145-151

19. Patrassi GM, Sartori MT, Viero ML, Scarano L, Boscaro M, Girolami A (1992) The fibrinolytic potential in patients with Cushing's disease: a clue to their hypercoagulable state. BloodCoagul Fibrinolysis 3:789-793

20. Arnaldi G, Mancini T, Polenta B, Boscaro M (2004) Cardiovascular risk in Cushing'ssyndrome. Pituitary 7:253-256

21. Brotman DJ, Girod JP, Posch A, et al. (2006) Effects of shortterm glucocorticoids on hemostatic factors in healthy volunteers. Thromb Res 118:247-252 
22. Jorgensen KA, Sorensen P, Freund L (1982) Effect of glucocorticosteroids on some coagulation tests. Acta Haematol 68:3942

23. Isacson S (1970) Effect of prednisolone on the coagulation and fibrinolytic systems. Scand J Haemat 7:212-216

24. Jilma B, Cvitko T, Winter-Fabry A, Petroczi K, Quehenberger P, Blann AD (2005) High dose dexamethasone increases circulating P-selectin and von Willebrand factor levels in healthy men. Thromb Haemost 94:797-801

25. Libby P, Simon DI (2001) Inflammation and thrombosis: the clot thickens. Circulation 103:1718-1720

26. Jensen BA, Sanders S, Frolund B, Hjortrup A (1988) Adrenocortical function in old age as reflected by plasma cortisol and ACTH test during the course of acute myocardial infarction. Arch Gerontol Geriatr 7:289-296

27. Otte C, Marmar CR, Pipkin SS, Moos R, Browner WS, Whooley MA (2004) Depression and 24-hour urinary cortisol in medical outpatients with coronary heart disease: The Heart and Soul Study. Biol Psychiatry 56:241-247

28. Kop WJ (1999) Chronic and acute psychological risk factors for clinical manifestations of coronary artery disease. Psychosom Med 61:476-487

29. Panagiotakos DB, Pitsavos C, Chrysohoou C, Tsetsekou E, Papageorgiou C, Christodoulou G, Stefanadis C (2004) Inflammation, coagulation, and depressive symptomatology in cardiovascular disease-free people; the ATTICA study. Eur Heart J 25:492-499

30. Doulalas AL, Rallidis LS, Gialernios T, et al. (2006) Association of depressive symptoms with coagulation factors in young healthy individuals. Atherosclerosis 186:121-125

31. Lahlou-Laforet K, Alhenc-Gelas M, et al. (2006) Relation of depressive mood to plasminogen activator inhibitor, tissue plasminogen activator, and fibrinogen levels in patients with versus without coronary heart disease. Am J Cardiol 97:1287-1891

32. Kop WJ, Hamulyak K, Pernot C, Appels A (1998) Relationship of blood coagulation and fibrinolysis to vital exhaustion. Psychosom Med 60:352-358

33. von Känel R, Maly FE, Frey K, Fischer JE (2003) Contribution of the type 1 plasminogen activator inhibitor $4 \mathrm{G} / 5 \mathrm{G}$ gene polymorphism to impaired fibrinolysis in vital exhaustion. Ital Heart $\mathbf{J}$ 4:791-796

34. Orth-Gomer K, Mittleman MA, Schenck-Gustafsson K, et al. (1997) Lipoprotein(a) as adeterminant of coronary heart disease in young women. Circulation 95:329-334

35. Myocardial Infarction Community Registers. (1976) Results of WHO international collaborative study. World Health Organisation Regional Office for Europe, Copenhagen, Denmark

36. Gillum RF, Fortmann SP, Prineas RJ, Kottke TE (1984) International diagnostic criteria for acute myocardial infarction and acute stroke. Am Heart J 108:150-158

37. Braunwald E (1989) Unstable angina. A classification. Circulation 80:410-414

38. Appels A, Hoppener P, Mulder P (1987) A questionnaire to assess premonitory symptoms of myocardial infarction. Int $\mathrm{J}$ Cardiol 17:15-24

39. Appels A, Falger PR, Schouten EG (1993) Vital exhaustion as risk indicator for myocardial infarction in women. J Psychosom Res 37:881-890

40. Appels A, Kop W, Bar F, de Swart H, Mendes de Leon C (1995) Vital exhaustion, extent ofatherosclerosis, and the clinical course after successful percutaneous transluminal coronary angioplasty. Eur Heart J 16:1880-1885
41. Hawthorne G, Elliott $P$ (2005) Imputing cross-sectional missing data: comparison of common techniques. Aust NZJ Psychiatry 39:583-590

42. Pearlin LI, Lieberman MA, Menaghan EG, Mullan JT (1981) The stress process. J Health Soc Behav 22:337-356

43. van Diest R, Appels A (1991) Vital exhaustion and depression: a conceptual study. J Psychosom Res 35:535-544

44. Kudielka BM, von Känel R, Gander ML, Fischer JE (2004) The interrelationship of psychosocial risk factors for coronary artery disease in a working population: do we measure distinct or overlapping psychological concepts?. Behav Med 30:35-43

45. Pruessner JC, Wolf OT, Hellhammer DH, et al. (1997) Free cortisol levels after awakening: areliable biological marker for the assessment of adrenocortical activity. Life Sci 61:2539-2549

46. Morrissey JH, Macik BG, Neuenschwander PF, Comp PC (1993) Quantitation of activated factor VII levels in plasma using a tissue factor mutant selectively deficient in promotingfactor VII activation. Blood 81:734-744

47. Vermylen C, de Vreker R, Verstraete M (1963) A rapid enzymatic method for assay offibrinogen fibrin polymerization time (FPT test). Clin Chim Acta 8:418-424

48. Jungner L, Walldius G, Holme I, Kolar W, Steiner E (1992) Apolipoprotein B and A-I inrelation to serum cholesterol and triglycerides in 43,000 Swedish males and females. Int J Clin Lab Res 21:247-255

49. Trinder $P$ (1969) Determination of glucose in blood using glucose oxidase with an alternative oxygen acceptor. Ann Clin Biochem 6:24-27

50. Hedman M, Nilsson E, de la Torre B (1989) Low sulpho-conjugated steroid hormone levels in systemic lupus erythematosus (SLE). Clin Exp Rheumatol 7:583-588

51. de la Torre B, von Krogh G, Svensson M, Holmberg V (1997) Blood cortisol and dehydroepiandrosterone sulphate (DHEAS) levels and CD4 T cell counts in HIV infection. Clin Exp Rheumatol 15:87-90

52. Cohen J (1988) Statistical power analysis for the behavioral sciences, 2nd edn. Erlbaum, Hillsdale NJ

53. Baron RM, Kenny DA (1986) The moderator-mediator variable distinction in social psychological research: conceptual, strategic, and statistical considerations. J Pers Soc Psychol 51:1173-1182

54. Kudielka BM, Bellingrath S, Hellhammer DH (2006) Cortisol in burnout and vital exhaustion an overview. G Ital Med Lav Erg 28(Suppl Psicologia 1):34-42

55. Alessi MC, Juhan-Vague I (2004) Contribution of PAI-1 in cardiovascular pathology. Arch Mal Coeur Vaiss 97:673-678

56. von Känel R, Kudielka BM, Abd-el-Razik A, Gander ML, Frey K, Fischer JE (2004) Relationship between overnight neuroendocrine activity and morning haemostasis in working men. Clin Sci 107:89-95

57. Princen HM, Moshage HJ, de Haard HJ, van Gemert PJ, Yap SH (1984) The influence of glucocorticoid on the fibrinogen messenger RNA content of rat liver in vivo and in hepatocyte suspension culture. Biochem J 220:631-637

58. Eller NH, Netterstrom B, Allerup P (2005) Progression in intima media thickness - the significance of hormonal biomarkers of chronic stress. Psychoneuroendocrinology 30:715-723

59. Clow A, Thorn L, Evans P, Hucklebridge F (2004) The awakening cortisol response:methodological issues and significance. Stress 7:29-37

60. Koenig W (2001) Inflammation and coronary heart disease: an overview. Cardiol Rev 9:31-35 